\title{
Resonance Scattering of Elastic Waves by an Elastic Inclusion in an Elastic Matrix: Numerical Calculations
}

\author{
GUILLERMO C. GAUNAURD, SENIOR MEMBER, IEEE, AND WILLIAM H. WERTMAN
}

\begin{abstract}
The scattering of an incident plane ultrasonic (longitudinal) wave by an elastic spherical inhomogeneity contained within an elastic matrix is studied. The emphasis is placed on the computation and analysis of basic multipole cases that result when different material behaviors are present in the matrix and in the inclusion. These calculations are useful in underwater acoustic applications. The above-mentioned behaviors are dominated by the soft or rigid backgrounds of the resonance scattering theory (RST). The first three-multipole coefficients appearing in the expansions for the total elastodynamic fields developed around the inhomogeneity during the scattering process, have been calculated in suitable frequency bands in all the cases considered. The examination of the modulus, the real parts, and the imaginary parts of these (complex) coefficients under the RST approach allows the quantitative assessment of the conditions under which monopole or dipole resonances will occur first, and the determination of the sizes of their relative magnitudes. The decomposition of the multipole coefficients into their resonance and background portions shows that it is the upward frequency shift of the background curves (a shift that occurs when the inclusions become stiffer than the matrix) that controls the dominance of either radial (monopole) or translation (dipole) oscillations of the inclusion. This has an effect on the dispersion curves of the composite that develop optical as well as acoustical branches. The real and imaginary parts of the multipole coefficients are respectively proportional to the attenuation and the effective wave speed in this simple inhomogeneous composite. In view of their physical significance, these quantities are displayed in all cases.
\end{abstract}

\section{INTRODUCTION}

$\mathrm{T}$ HE SCATTERING of elastic (longitudinal and transverse) waves by a spherical solid or fluid inhomogeneity contained within an elastic material is a difficult but quite basic problem with a lengthy history. The classical analysis started with Ying and Truell [1] and Pao and Mow [2]. This early work was later summarized in general monographs [3], [4]. With the advent of the resonance scattering theory (RST) [5], this problem was reexamined in much greater detail. A review of this work has appeared recently [6] that summarizes most of the classical and RST literature on the subject and analyzes all the particular cases that can be extracted from it. Even though the complete resonance scattering approach to be used by us in the present work as applied to a single elastic [6],

Manuscript received February 12,1987; revised August 7, 1987. This work was supported in part by the Independent Research Board of the $\mathrm{Na}$ val Surface Weapons Center and in part by the Office of Naval Research.

The authors are with the Naval Surface Weapons Center, White Oak Laboratory, Silver Spring, MD 20903-5000.

IEEE Log Number 8820906
[7] or fluid [8] spherical inhomogeneity within an elastic matrix has appeared already [6], it is its application to a novel case with special uses and implications that motivates the present work.

A brief summary of the findings of the RST as it applies to a fluid/elastic inclusion within an elastic matrix can be given as follows. Whenever an incident elastic wave falls upon an inclusion, the inclusion is invited to oscillate with all the frequencies present in the incident wave. The inclusion will, however, naturally respond vibrating only with that particular subset of frequencies that is also present in its own resonance spectrum. The RST consists of a series of steps that ultimately isolate the inclusion's resonances, as they appear buried within the active echo that the inclusion returns. This echo can be viewed as a characteristic signature, in frequency or time, of the type of inclusion that generated it. Thus RST is a potential targetidentification tool. The first step of RST models the scatterer as penetrable, with interior fields coupled to the outer fields through suitable boundary conditions at the inclusion's surface. The second step requires an analysis of each one of the modes or partial-waves that make up the inclusion's (summed) cross section. Finally, the third step separates the two basic contributions contained with each mode. These are in the form of smooth backgrounds and a superimposed set of discrete spiky resonances. The backgrounds, in the terminology of the method, depend on the inclusion's shape, but assume it to be impenetrable (viz., rigid or soft). The resonances are a consequence of the penetrable (viz., elastic) composition of the inclusion, and they manifest themselves as discrete dips/peaks, riding superimposed on the shape-dependent smooth backgrounds. The resonances correspond to the penetration of energy into the inclusion, which only occurs through discrete spectral windows centered at the inclusion's resonance frequencies. For inclusions with nonseparable shapes, the decomposition of the scattering amplitude or the scattering cross section into modal or partial-wave contributions is not possible, and the portions corresponding to backgrounds and resonances can only be separated from each other in the summed cross section, instead of in each of its partial-wave constituents. The separation between modal backgrounds and resonances which was described previously, can be used to isolate the resonance 
spectrum of any inclusion. This is done by a process of suitable background suppression from the received elastic echo. Specific examples of this resonance isolation process have been given before [5]. Remarkably, this process can be carried on not only theoretically [5], [8], but experimentally as well [9]. The main purpose of this paper is to study the hierarchy of multipole occurrences for various pertinent combination of substances, and to establish the ordering of multipole resonances for some important matrix/inclusion extremes of elastic behavior.

\section{Theoretical Background}

Very briefly, if an incident longitudinal plane elastic wave described by the Debye potentials

$$
\begin{aligned}
& \phi_{\text {inc }}^{(1)}=\phi_{0} e^{-i \omega t} \sum_{n=0}^{\infty} i^{n}(2 n+1) j_{n}\left(k_{d_{1}} r\right) P_{n}(\cos \theta) \\
& \Phi_{\text {inc }}^{(1)}=0
\end{aligned}
$$

is incident on a spherical elastic inclusion, the total elastic field around it is given by the total elastic potentials

$$
\begin{aligned}
\phi_{\mathrm{tot}}^{(1)}= & \phi_{\mathrm{inc}}^{(1)}+\phi_{\mathrm{sc}}^{(1)}=\phi_{0} e^{-i \omega t} \sum_{n=0}^{\infty} i^{n}(2 n+1)\left[j_{n}\left(k_{d_{1}} r\right)+\right. \\
& \left.+A_{n} h_{n}^{(1)}\left(k_{d 1} r\right)\right] P_{n}(\cos \theta), \\
\psi_{\mathrm{tot}}^{(1)}= & \phi_{0} e^{-i \omega t} \sum_{n=0}^{\infty} i^{n}(2 n+1) B_{n} h_{n}^{(1)}\left(k_{s 1} r\right) P_{n}(\cos \theta) \\
= & \psi_{\mathrm{inc}}^{(1)}+\psi_{\mathrm{sc}}^{(1)}=\psi_{\mathrm{sc}}^{(1)},
\end{aligned}
$$

where $A_{n}$ and $B_{n}$ are coefficients determined from the boundary conditions. For elastic inclusions they are given by quotients of $4 \times 4$ determinants such as

$$
\begin{aligned}
& A_{n}=-\frac{\left|\begin{array}{llll}
\operatorname{Re} d_{11} & d_{12} & d_{13} & d_{14} \\
\operatorname{Re} d_{21} & d_{22} & d_{23} & d_{24} \\
\operatorname{Re} d_{31} & d_{32} & d_{33} & d_{34} \\
\operatorname{Re} d_{41} & d_{42} & d_{43} & d_{44}
\end{array}\right|}{\left|\begin{array}{llll}
d_{11} & d_{12} & d_{13} & d_{14} \\
d_{21} & d_{22} & d_{23} & d_{24} \\
d_{31} & d_{32} & d_{33} & d_{34} \\
d_{41} & d_{42} & d_{43} & d_{44}
\end{array}\right|} \\
& B_{n}=-\frac{\left|\begin{array}{llll}
d_{11} & \operatorname{Re} d_{11} & d_{13} & d_{14} \\
d_{21} & \operatorname{Re} d_{21} & d_{23} & d_{24} \\
d_{31} & \operatorname{Re} d_{31} & d_{33} & d_{34} \\
d_{41} & \operatorname{Re} d_{41} & d_{43} & d_{44}
\end{array}\right|}{\left|\begin{array}{llll}
d_{11} & d_{12} & d_{13} & d_{14} \\
d_{21} & d_{22} & d_{23} & d_{24} \\
d_{31} & d_{32} & d_{33} & d_{34} \\
d_{41} & d_{43} & d_{34} & d_{44}
\end{array}\right|}
\end{aligned}
$$

where all the elements $d_{i j}$ are given in the Appendix and elsewhere [6], [7]. In general, the relation between the elastic displacement field $\vec{u}$ and the three scalar Debye potentials $(\phi, \psi, \chi)$ is

$$
\vec{u}=\vec{\nabla} \phi+\vec{\nabla} \times[\vec{\nabla} \times(\vec{r} \psi)+\vec{\nabla} \times(\vec{\nabla} \times \vec{r} \chi)] .
$$

In this particular problem, however, $\chi \equiv 0$. Further details can be found elsewhere [6]. If the inclusion is fluid in composition, then the coefficients $A_{n}$ and $B_{n}$ are ratios of two $3 \times 3$ determinants [5], [8]. All the possible degenerate cases have been considered and analyzed under the RST approach in our recent review [6]. Some of the most pertinent (normalized) form-functions $f_{\infty}^{p p}$ and $f_{\infty}^{p s}$ in the backscattering direction $\theta=\pi$, are given by

$$
\begin{aligned}
& \frac{1}{a} f_{\infty}^{p p}(\pi)=\frac{1}{a} \sum_{n=0}^{\infty} f_{n}^{p p}(\pi)=\frac{1}{i x_{d_{1}}} \sum_{n=0}^{\infty}(-1)^{n}(2 n+1) A_{n} \\
& \frac{1}{a} f_{\infty}^{p p}(\pi)=\frac{1}{a} \sum_{n=0}^{\infty} f_{n}^{p s}(\pi)=\frac{1}{i x_{s 1}} \sum_{n=0}^{\infty}(-1)^{n}(2 n+1) B_{n}
\end{aligned}
$$

where $f_{\infty}^{p p}$ and $f_{\infty}^{p s}$ are form functions corresponding to the cases of an incident compressional $(p)$ wave and either a scattered $p$ or $s$ (shear) wave, respectively. It is often convenient to plot some of the partial waves $(n=0,1,2$, ...) contained within the form functions. We select to display the proportional quantities

$$
\begin{aligned}
& k_{d_{1}} f_{n}^{p p}(\pi)=(-1)^{n}(2 n+1) A_{n} / i \\
& k_{s 1} f_{n}^{p s}(\pi)=(-1)^{n}(2 n+1) B_{n} / i
\end{aligned}
$$

where $x_{d_{1}} \equiv k_{d_{1}} a, x_{s !} \equiv k_{s !} a$, and $n=0,1,2, \cdots$. In view of the relations:

$$
x_{s_{1}}=\frac{c_{d_{1}}}{c_{s_{1}}} x_{d_{1}} \quad x_{d_{2}}=\frac{c_{d_{1}}}{c_{d_{2}}} x_{d_{1}} \quad x_{s_{2}}=\frac{c_{d_{1}}}{c_{s_{2}}} x_{d_{1}}
$$

the coefficients $A_{n}$ and $B_{n}$ can be all expressed in terms of $x_{d_{1}}$. They are, in fact, analytic functions of $x_{d_{1}}$ and $n$, if these were to be considered as real variables. Plotting (6) rather than (5) has the advantage of having all the resonance features of about the same size, since the factors $x_{d_{1}}$ and $x_{s \mid}$ have been transfered to the left sides. The first few of these coefficients are quite important, and we have labored extensively [6]-[8] in the past to find analytic expressions for the first few, and to explain all their terms in detail. We note that coefficients $A_{n}$ and $B_{n}$ are complex and so are the partial waves $f_{n}^{p p}$ and $f_{n}^{p s}$ contained within the scattering amplitudes. Since the $A_{n}$ and $B_{n}$ are divided by $i=\sqrt{-1}$, the real (imaginary) parts of these coefficients are the imaginary (real) parts of $f_{n}^{p p}$ and $f_{n}^{p s}$. The real and imaginary parts of $f_{n}^{p p}$ (and $f_{n}^{p s}$ ) are associated with the wave speed and the attenuation of the scattered waves, respectively. Sometimes it is convenient to plot the moduli $\left|A_{n}\right|,\left|B_{n}\right|$, and at others it is best to look at $\operatorname{Re} A_{n}, \operatorname{Im} A_{n}$, or Re $B_{n}, \operatorname{Im} B_{n}$.

Finally, the appropriate backgrounds of the RST can be subtracted from the partial waves to isolate the resonances 
within each normal mode. Soft or rigid backgrounds are possible, depending on the relative compositions of matrix and inclusion. Therefore, for tenuous inclusions (soft background case) one has

$$
\begin{aligned}
k_{d_{1}} f_{\infty}^{(\mathrm{res}) p p}(\pi) & =k_{d_{1}} \sum_{n=0}^{\infty} f_{n}^{(\mathrm{res}) p p}(\pi) \\
& =\sum_{n=0}^{\infty}(-1)^{n}(2 n+1)\left[A_{n}-A_{n}^{\text {(soft })}\right] / i \\
k_{s 1} f_{\infty}^{\text {(res) } p s}(\pi) & =k_{s_{1}} \sum_{n=0}^{\infty} f_{n}^{\text {(res)ps }}(\pi) \\
& =\sum_{n=0}^{\infty}(-1)^{n}(2 n+1)\left[B_{n}-B_{n}^{(\text {soft })}\right] / i
\end{aligned}
$$

while for very heavy inclusions (rigid background case) we find

$$
\begin{aligned}
k_{d_{1}} f_{\infty}^{\text {(res) } p p}(\pi) & =k_{d_{1}} \sum_{n=0}^{\infty} f_{n}^{(\text {res }) p p}(\pi) \\
& =\sum_{n=0}^{\infty}(-1)^{n}(2 n+1)\left[A_{n}-A_{n}^{\text {(rig) }}\right] / i \\
k_{s 1} f_{\infty}^{\text {(res) ps }}(\pi) & =k_{s_{1}} \sum_{n=0}^{\infty} f_{n}^{\text {(res) ps }(\pi)} \\
& =\sum_{n=0}^{\infty}(-1)^{n}(2 n+1)\left[B_{n}-B_{n}^{\text {(rig) }}\right] / i
\end{aligned}
$$

The pertinent "backgrounds" to be used above are ratios of two $2 \times 2$ determinants as follows:

$$
A_{n}^{\text {(soft) }}=-\frac{\left|\begin{array}{ll}
\operatorname{Re} d_{31} & d_{32} \\
\operatorname{Re} d_{41} & d_{42}
\end{array}\right|}{\left|\begin{array}{ll}
d_{31} & d_{32} \\
d_{41} & d_{42}
\end{array}\right|} \quad B_{n}^{\text {(soft) }}=-\frac{\left|\begin{array}{ll}
d_{31} & \operatorname{Re} d_{31} \\
d_{41} & \operatorname{Re} d_{41}
\end{array}\right|}{\left|\begin{array}{ll}
d_{31} & d_{32} \\
d_{41} & d_{42}
\end{array}\right|}
$$

for the soft case, and

$$
A_{n}^{\text {(rig) }}=-\frac{\left|\begin{array}{ll}
\operatorname{Re} d_{11} & d_{12} \\
\operatorname{Re} d_{21} & d_{22}
\end{array}\right|}{\left|\begin{array}{ll}
d_{11} & d_{12} \\
d_{21} & d_{22}
\end{array}\right|} \quad B_{n}^{\text {(rig) }}=-\frac{\left|\begin{array}{ll}
d_{11} & \operatorname{Re} d_{11} \\
d_{31} & \operatorname{Re} d_{21}
\end{array}\right|}{\left|\begin{array}{ll}
d_{11} & d_{12} \\
d_{21} & d_{22}
\end{array}\right|}
$$

for the rigid case. All the elements $d_{i j}$ are given in the Appendix. If the inclusion is fluid, the $4 \times 4$ determinants reduce to order $3 \times 3$ in (3) and (4). The backgrounds in (10) and (11) then reduce to quotients of single elements.
These cases have been discussed elsewhere [6], [8], [5]. Further details can be found in [6].

\section{A Specific Application}

Decouplers and sound-absorbing materials for underwater applications often consist of nonmetallic substances containing various types of inclusions. It is not uncommon to use spherical inclusions. If we consider a single such inhomogeneity in the matrix, it is possible to use the above formulation to evaluate the first few coefficients given in Section II. The first three such coefficients, viz., $A_{0}, A_{1}$, and $A_{2}$, are the most useful. These correspond to the monopole, dipole, and quadrupole modes of vibration, respectively. The monopole mode describes the inclusion's oscillations breathing in and out, in the radial direction. The dipole mode describes back-and-forth rigidbody translational motions of the inclusion within the matrix, and the quadrupole is associated with more complex oscillations permitting surface deformations. For matrix/ inclusion compositions for which the soft background is the appropriate one, the fundamental monopole peak occurs earlier than the fundamental peak of the dipole or of any higher order multipole. For composition combinations of matrix and inclusion for which the rigid background is the appropriate one, the fundamental peak of the dipole is the one that occurs before all other fundamental peaks of all other multipoles. Even though in this case the dipole peak appears first, the amplitude of the monopole peak is still the largest. The above statements can be verified with the example of either a metallic inclusion in a nonmetallic matrix (rigid background case), or of a nonmetallic inclusion in a metal matrix (soft background case). In this paper, we will consider the case of a steel sphere in an epoxy matrix, or an epoxy sphere in a steel matrix to illustrate these points.

\section{Numerical Results}

The material parameters of all the substances discussed in the following are given in Table I. Medium 1 is the matrix and medium 2 is the inclusion.

Fig. 1 displays the moduli $\left|A_{n}\right|$ versus $k_{d !} a$ for $n=0$, 1 , and 2 in the range $0.1 \leq k_{d_{1}} a \leq 10$, for an epoxy sphere in a steel matrix (soft background case). The plot of $\left|A_{0}\right|$ exhibits the fundamental monopole peak that has the largest amplitude and appears at the lowest frequency when compared to the other multipole coefficients. Thus the giant monopole peak is the first and the strongest of all three coefficients $A_{0}, A_{1}$, and $A_{2}$.

Figs. 2 and 3 display the plots of $\operatorname{Re}\left[-A_{n}\right]$ and $\operatorname{Im}$ $\left[-A_{n}\right]$ for $n=0,1,2$, for the same epoxy sphere in a steel matrix. These graphs are associated, respectively, with the effective wavenumber (or wave speed) and the effective attenuation in this simple inhomogeneous composite containing just a single inclusion. In both these figures we see the amplitude dominance of the monopole peak (solid line), and its first occurrence as the frequency increases. We remark that the monopole fundamental "resonance" is made up of two portions. One comes from 
TABLE I

\begin{tabular}{lcccrr}
\hline Material & $\rho\left(\mathrm{g} / \mathrm{cm}^{3}\right)$ & $c_{d}(\mathrm{~cm} / \mathrm{s})$ & $c_{s}(\mathrm{~cm} / \mathrm{s})$ & $\lambda\left(\right.$ dyne $\left./ \mathrm{cm}^{2}\right)$ & $\mu\left(\mathrm{dyne} / \mathrm{cm}^{2}\right)$ \\
\hline Steel & 7.84 & $5.80 \times 10^{5}$ & $3.10 \times 10^{5}$ & $113 \times 10^{10}$ & $75 \times 10^{10}$ \\
Epoxy & 1.18 & $2.54 \times 10^{5}$ & $1.16 \times 10^{5}$ & $4.44 \times 10^{10}$ & $1.59 \times 10^{10}$ \\
Glass & 2.492 & $5.28 \times 10^{5}$ & $3.24 \times 10^{5}$ & $17.2 \times 10^{10}$ & $26 \times 10^{10}$ \\
\hline$c_{d}=[(\lambda+2 \mu) / \rho]^{1 / 2} ; c_{s}=(\mu / \rho)^{1 / 2} ; \lambda=\rho\left(c_{d}^{2}-2 c_{s}^{2}\right) ; \mu=\rho c_{s}^{2}$.
\end{tabular}

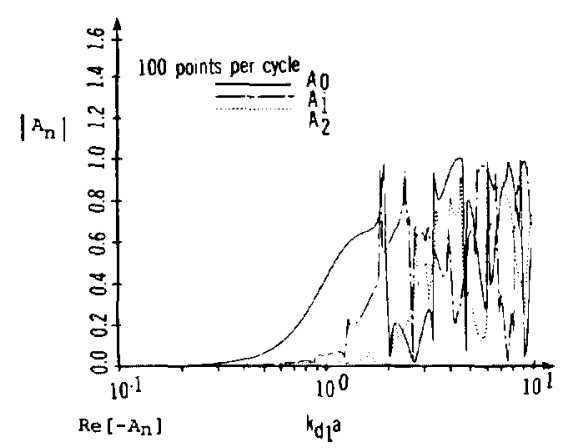

Fig. 1. Modulus of $A_{n}$ versus $k_{d_{1}} a$ for epoxy sphere in steel matrix ( $n=$ 0,1 , and 2 ). (from (3)).

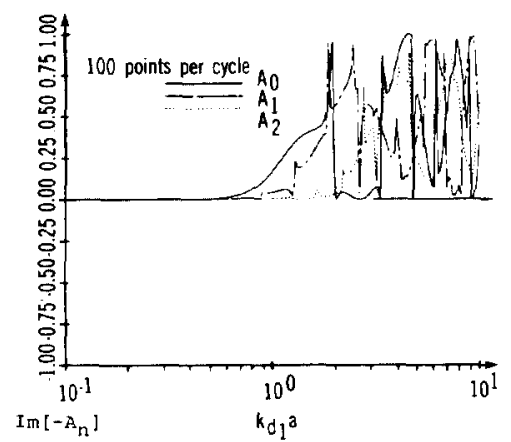

Fig. 2. $\operatorname{Re}\left[-A_{n}\right]$ versus $k_{d_{1}} a$ for epoxy sphere in steel matrix ( $n=0,1$, and 2 ).

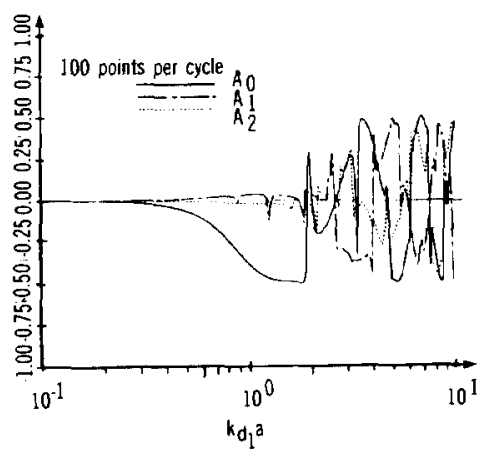

Fig. 3. Im $\left[-A_{n}\right]$ versus $k_{d !} a$ for epoxy sphere in steel matrix ( $n=0,1$, and 2$)$.

the "background," and the other from the resonances "proper." We have studied these portions before [8] for air bubbles in water, fluid-filled cavities in rubbers, and similar configurations for which the soft background is the one that isolates the resonances. If the combination of compositions in the matrix and the inclusion changes, so that the problem's background moves away from the soft one and toward the rigid one [10, Fig. 8], then the first peak will not be as large and will belong more to $A_{1}$ than to $A_{0}$. We will return to this later. The RST has repeatedly demonstrated [5], [6] that the resonances are discrete spiky features riding on top of usually smooth, continuous backgrounds. Fig. 2 for $\operatorname{Re}\left[-A_{0}\right]$ shows the edge of the first background lobe, extending to low-frequencies down to values of $k_{d_{1}} a \approx 0.5$. In this case, the fundamental monopole resonance occurs at $k_{d_{1}} a \approx 2$. The curves corresponding to the higher order multipoles, viz., $A_{1}$ and $A_{2}$, are seen to exhibit some weaker resonance features in the region $0.5 \leq k_{d_{1}} a \leq 2$.

We now consider the case of a steel sphere in an epoxy matrix, which corresponds to the rigid background case. Fig. 4 displays the modulus of the first three multipole coefficients (viz., $\left|A_{n}\right|$ ) for $n=0,1$, and 2 . The graph of $\left|A_{0}\right|$ (solid line) exhibits its first peak at $k_{d_{1}} a \approx 3$, but this is not the first one that occurs since the dipole coefficient $\left|A_{1}\right|$ is seen to show a small peak at $k_{d_{1}} a \approx 0.4$. We will see later that these peaks are really caused by the first background lobes, rather than by multipole fundamental resonances. Figs. 5 and 6 are analogous to Figs. 2 and 3, but now for the steel sphere in the epoxy matrix (rigid background case). Figs. 5 and 6, respectively, exhibit three curves for $\operatorname{Re}\left[-A_{n}\right]$ and $\operatorname{Im}\left[-A_{n}\right]$ for $n=$ 0,1 , and 2. The curve for $\operatorname{Re}\left[-A_{0}\right]$ continues to have the highest amplitude, but now the curve $\operatorname{Re}\left[-A_{1}\right]$ for the dipole coefficient, develops its small peak first. This behavior is the norm for any situation in which the rigid background is the one that isolates the resonances. It follows that the breathing-type radial oscillations of the monopole mode can be excited with a lot more ease and strength when the inclusion is soft or compliant relative to the matrix. When the inclusion is a fluid (viz., a gas), these radial oscillations, particularly the first one, are gigantic [11] and dwarf all others. However, for inclusions rigid or stiff compared to a softer matrix, the radial monopole oscillations are harder to excite than the backand-forth rigid-body translational oscillations typical of the dipole motion. Although these are weaker than those of the monopole, in this case they tend to appear at lower frequencies.

Thus far we have not taken any advantage of the decomposition of these coefficients $A_{n}$ into "background" and "resonance" portions, in the manner typical of the RST [5], [6]. We spoke above of the "monopole resonance peak," when we really should have referred to the background contribution to the curve associated with the monopole coefficient $A_{0}$. To clarify these important distinctions, we split the three coefficients $A_{n}(n=0,1,2)$ 


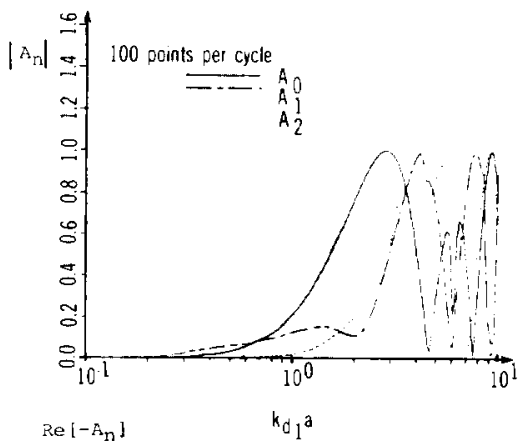

Fig. 4. Modulus of $A_{n}$ versus $k_{d_{1}}$ a for steel sphere in epoxy matrix ( $n=$ 0,1 , and 2 ).

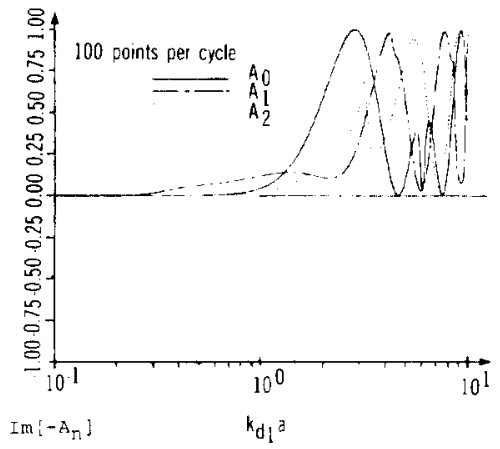

Fig. 5. Re $\left[-A_{n}\right]$ versus $k_{d_{1}} a$ for steel sphere in epoxy matrix $(n=0,1$, and 2).

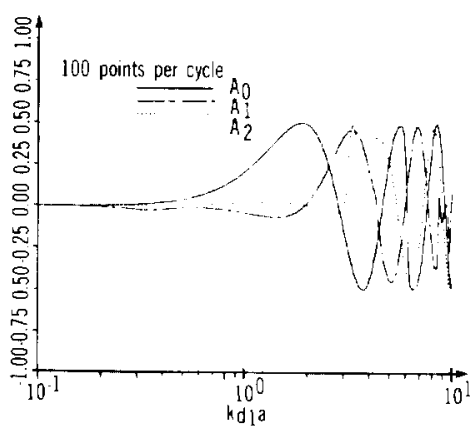

Fig. 6. Im $\left[-A_{n}\right]$ versus $k_{d_{1}} a$ for steel sphere in epoxy matrix $(n=0,1$, and 2 ).

into background and resonance portions in the usual manner of the RST. This will decompose each one of the three quantities $\left|A_{n}\right|, \operatorname{Re}\left[-A_{n}\right]$, and $\operatorname{Im}\left[-A_{n}\right]$, displayed in the above figures for $n=0,1$, and 2 , into two portions each. Since each one of these three quantities has different physical meaning and interpretation (viz., they pertain, respectively, to the modal scattering amplitude, effective attenuation, and effective wavenumber or wave speed) it is informative to decompose all three. The resonance portions of the multipole coefficients are given by (8) and (9), and the appropriate rigid or soft backgrounds, by (10), (11).

For an epoxy sphere in a steel matrix (soft background case), Figs. 7 and 8 separate the background and the resonance portions contained within the $\left|A_{n}\right|$ in Fig. 1. Thus Fig. 7 pertains to $\left|A_{n}^{\text {(soft) }}\right|$ and Fig. 8 to $\left|A_{n}-A_{n}^{\text {(soft) }}\right|$. Fig. 9 corresponds to $-\operatorname{Re}\left[A_{n}^{\text {(soft) }}\right]$ and Fig. 10 to $-\operatorname{Re}$

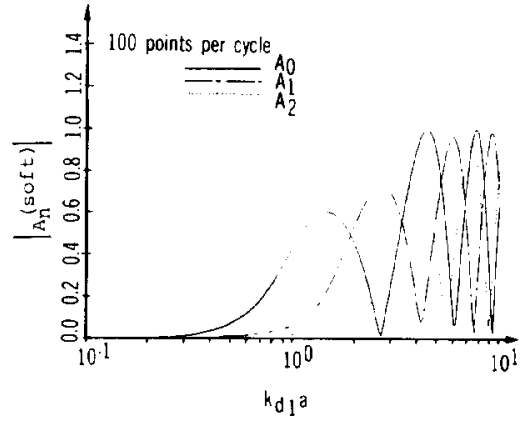

Fig. 7. Soft background $\left|A_{n}^{\text {(soft) }}\right|$ for epoxy inclusion in steel matrix versus $k_{d_{1}} a$ for $n=0,1$, and 2 (from (10)).

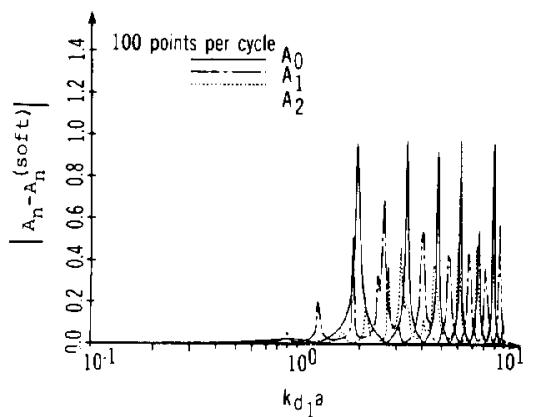

Fig. 8. Isolated resonances $\left|A_{n}-A_{n}^{\text {(soft) }}\right|$ for epoxy inclusion in steel matrix, versus $k_{d_{1}} a$, for $n=0,1$, and 2 (from (3) and (10)).

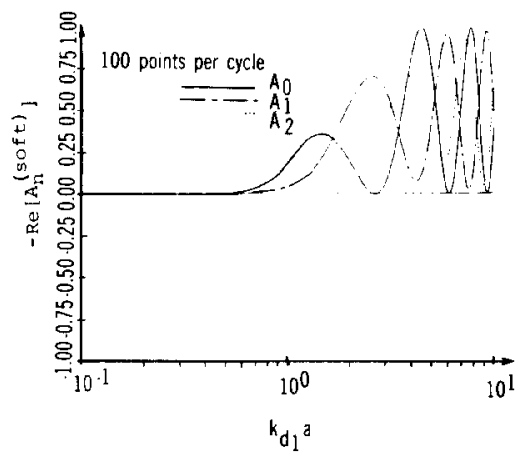

Fig. 9. Background absorption $-\operatorname{Re}\left[\boldsymbol{A}_{n}^{\text {(soft) }}\right]$ for epoxy inclusion in steel matrix, versus $k_{d_{1}} a$, for $n=0,1$, and 2 .

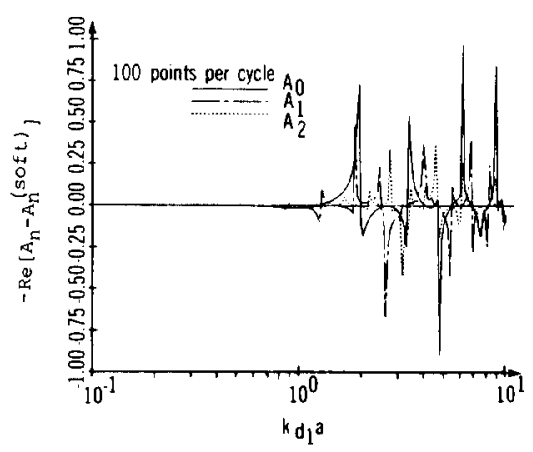

Fig. 10. Resonance contribution to absorption, viz., $-\operatorname{Re}\left[A_{n}-A_{n}^{\text {(suft) }}\right]$, for epoxy inclusion in steel matrix, versus $k_{d_{1}} a$, for $n=0,1$, and 2 .

$\left[A_{n}-A_{n}^{\text {(soft) }}\right]$. Fig. 11 pertains to $-\operatorname{Im}\left[A_{n}^{\text {(soft) }}\right]$ and Fig. 12 to $-\operatorname{Im}\left[A_{n}-A_{n}^{\text {(soff) }}\right]$. These pairs of quantities are all plotted versus $k_{d_{1}} a$ in the range $0.1 \leq k_{d_{1}} a \leq 10$ for $n=$ 0,1 , and 2 in Figs. 7-12. 


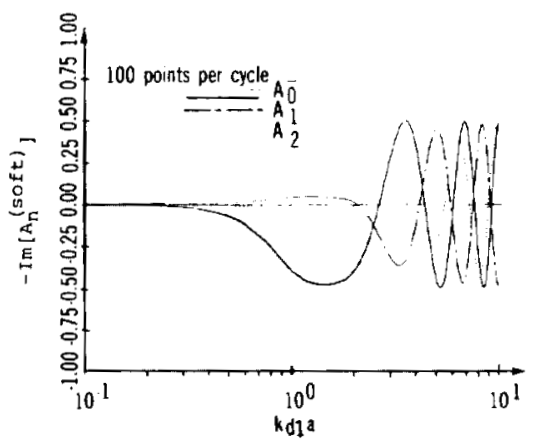

Fig. 11. Background contribution to effective wavespeed, viz., $-\mathrm{Im}$ $\left[A^{\text {lsofit }}\right]$, for epoxy inclusion in steel matrix, versus $k_{d} a$, for $n=0,1$, and 2 .

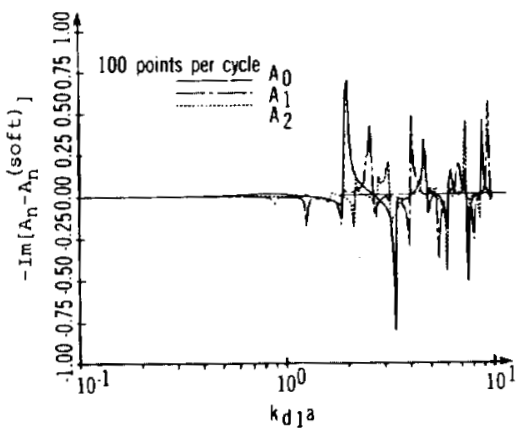

Fig. 12. Resonance contribution to effective wavespeed, viz., $-\operatorname{lm}\left[A_{n}-\right.$ $A_{n}^{\text {(sofft) }}$, for epoxy inclusion in steel matrix, versus $k_{d_{1}}$ for $n=0,1$, and

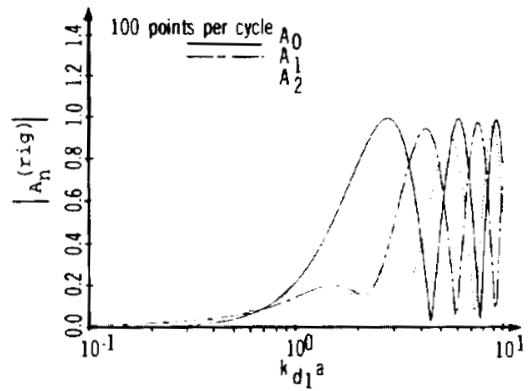

Fig. 13. Rigid background $\left|A^{\text {(nig) }}\right|$ for steel inclusion in epoxy matrix versus $k_{d,} a$, for $n=0,1$, and 2 . (from (11)).

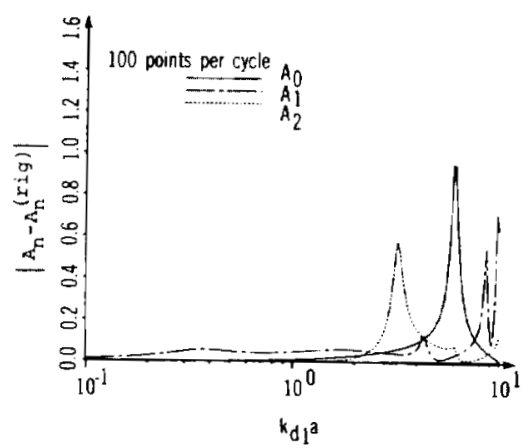

Fig. 14. Isolated resonances $\left|A_{n}-A_{n}^{\text {(tig) }}\right|$ for steel inclusion in epoxy matrix versus $k_{d, a} a$, for $n=0,1$, and 2 (from (3) and (9))

The same procedure can be followed for the case of a steel sphere in an epoxy matrix (rigid background case). Figs. 13 and 14 separate the background and resonance

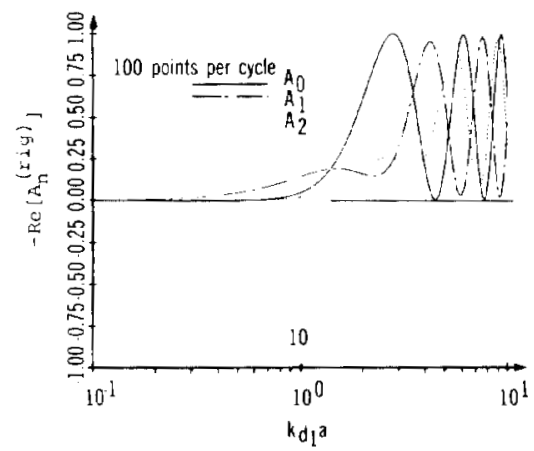

Fig. 15. Background component of absorption $-\operatorname{Re}\left[A_{n}^{\text {iriz) }}\right]$ for steel inclusion in epoxy matrix versus $k_{d i} a$, for $n=0,1$, and 2 .

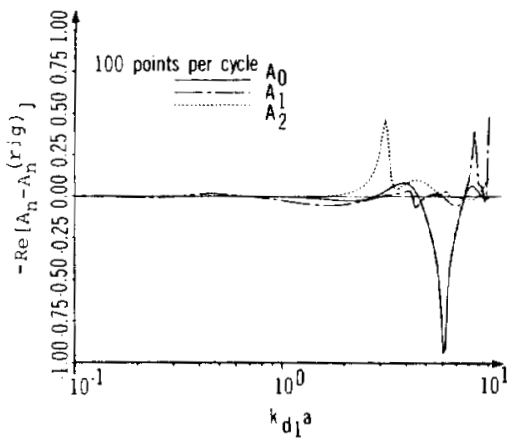

Fig. 16. Resonance contribution to absorption $-\operatorname{Re}\left[A_{n}-A_{n}^{(\text {rig) }}\right]$ for steel inclusion in epoxy matrix versus $k_{d,} a$, for $n=0,1$, and 2 .

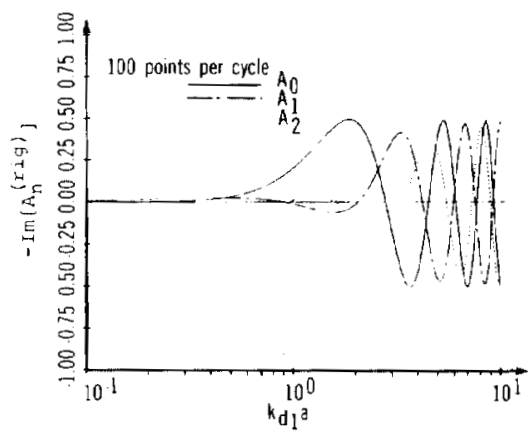

Fig. 17. Background contribution to effective wavespeed, viz., $-\mathrm{Im}$ $\left[A_{n}^{\text {(ing) }}\right]$, for steel inclusion in epoxy matrix versus $k_{d 1} a$, for $n=0,1$, and 2 .

portions contained within the $\left|A_{n}\right|$ in Fig. 4. Thus Fig. 13 corresponds to $\left|A_{n}^{\text {(rig) }}\right|$ and Fig. 14 to $\left|A_{n}-A_{n}^{\text {(rig) }}\right|$. Fig. 15 pertains to $-\operatorname{Re}\left[A_{n}^{\text {(rig) }}\right]$ and Fig. 16 to $-\operatorname{Re}\left[A_{n}\right.$ $\left.-A_{n}^{\text {(rig) }}\right]$. The quantities $-\operatorname{Im}\left[A_{n}^{\text {(rig) }}\right]$ and $-\operatorname{Im}\left[A_{n}-\right.$ $\left.A_{n}^{\text {(rig) }}\right]$, are, respectively, displayed in Figs. 17 and 18 . Again, the range of all the plots for the steel inclusion in epoxy is $0.1 \leq k_{d_{1}} a \leq 10$.

In earlier work [7, appendix] we once considered glass inclusions in an epoxy matrix. The material properties are given in Table I. The situation is very similar to a steel sphere in epoxy. The background is rigid. Fig. 19 shows $\left|A_{n}\right|$ versus $k_{d_{1}} a$ for $n=0,1,2$. Fig. 20 exhibits $\mathrm{Re}$ $\left[-A_{n}\right]$ versus $k_{d_{i}} a$ for $0.1 \leq k_{d_{1}} a \leq 10$. The first lobe of the dipole's $\left(A_{1}\right)$ background occurs before all others, but its magnitude is very small compared to $A_{0}$. This is the same situation as in Fig. 5. 


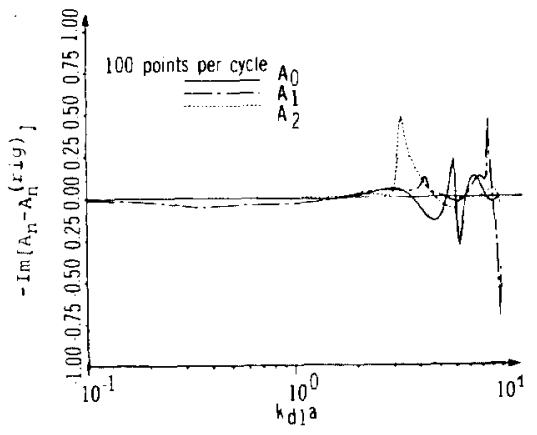

Fig. 18. Resonance contribution to effective wave speed viz., $-\operatorname{Im}\left[A_{n}-\right.$ $A_{n}^{\text {(rig) }}$, for steel inclusion in epoxy matrix versus $k_{d_{1}} a$, for $n=0,1$, and 2 .

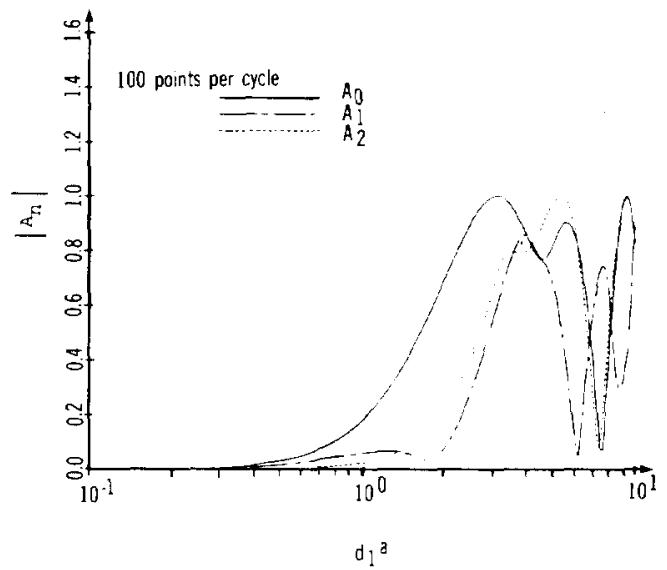

Fig. 19. Analogous to Fig. 4. Modulus of $A_{n}$ versus $k_{d,}$ a for glass spherical inclusion in epoxy matrix ( $n=0,1$, and 2 ). Rigid background case.

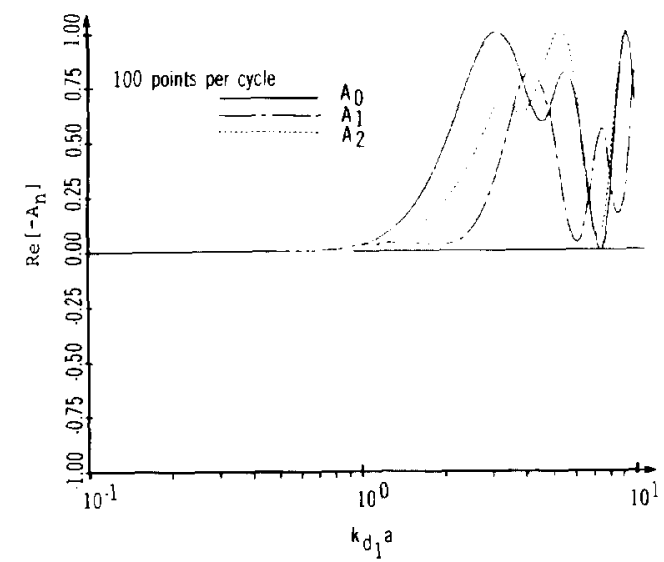

Fig. 20. Analogous to Fig. 5. Re $\left[-A_{n}\right]$ versus $k_{d} a$ for glass inclusion in epoxy matrix $(n=0,1$, and 2$)$. Total (background plus resonance) effective absorption.

\section{Physical interpretations and Discussion}

Resonances in the inclusion generate surface waves that revolve around it at the interface with the matrix. These waves are attenuated and diminish their amplitude as they shed energy in their geodesic circumnavigations. Even though both matrix and inclusion are elastic and thus contain no losses, energy losses take place through a modeconversion process in the matrix/inclusion system. There is an energy exchange between $f_{\infty}^{p p}$ and $f_{\infty}^{p s}$ (see (6)) due to scattering, which produces a loss without the presence of viscoelastic behavior, which accounts for attenuation in purely elastic materials. Recall that $\operatorname{Re}\left[A_{n}\right]$ and $\operatorname{Im}$ $\left[A_{n}\right]$ are, respectively, proportional to the $\operatorname{Im}\left[f_{n}^{p p}\right]$ and $\operatorname{Re}\left[f_{n}^{p p}\right]$ which are associated with the effective attenuation and wave speed in the composite (see (6)).

Decomposing the curves for the monopole, dipole, and quadrupole coefficients $\left(A_{0}, A_{1}, A_{2}\right)$ into their background and resonance portions, in the manner typical of the RST, brings out a number of interesting points. The first background lobe of $A_{0}$ for an epoxy sphere in steel appears before the first such lobe for the higher order multipoles. This may give the false impression that the monopole resonance always occurs first. This happens only for gas inclusions, for which the monopole resonance is giant [10], but not for solid inclusions, in which case it is not. As one can clearly see in Fig. 8 after the soft background has been suppressed, the first dipole and quadrupole resonances occur before that of the monopole, although they are smaller in amplitude. If one wishes to apply further details of the RST approach to examples such as the ones given here, one must be careful that the background/resonance decomposition has been accomplished. Only after the resonances have been isolated by subtracting the proper background of the case in question can one talk about true resonance locations and their widths $\Gamma_{n i}$.

The first lobe in the background curve of $A_{1}$ for a steel sphere in an epoxy matrix appears before all the first lobes of all the other multipole coefficients (see Fig. 4), However, this lobe is small in amplitude compared to the others. In this (steel in epoxy) case, both dipole and quadrupole coefficients have first (i.e., fundamental) resonances occurring before that of the monopole (Fig. 14). Thus the first monopole resonance appears after the first dipole and quadrupole resonances for steel in epoxy (Fig. 14). This is also true for epoxy in steel (Fig. 8). In both cases, the monopole resonance is much stronger in amplitude. It seems that only for gas inclusions [8], [10] the first monopole resonance occurs before the first resonance from the other higher order multipoles. It is only for gas inclusions that the first monopole resonance is giant and that it has reinforcing and coincident contributions coming from the background portion associated with the matrix wall, as well as from the resonance portion proper, associated with the inclusion's gas. As soon as the inclusion becomes solid, the contribution from the background diminishes and the background curve itself shifts away from the soft pattern (see Fig. 7) toward the rigid one (see Fig. 13). In view of this upward shift in frequency that misaligns previously reinforcing backgrounds and resonances, the first monopole resonance ceases to be giant, although it continues to be the largest. Furthermore, it then moves to a position after the first dipole and, many times, after the first quadrupole resonance. In spite of the upward shift in frequency discussed here as the inclusions become solid and stiffer, all the first (fundamental) resonances of all the cases studied here are quite small. They all occur within the Rayleigh region $(a / \lambda) \gtrless 1$. (or $\left.k_{d^{\prime} \mid} a \gtrless 2 \pi\right)$. 
The migration of the lobes in the background curves from the case of epoxy in steel (i.e., soft, see Fig. 7), to that of steel in epoxy (i.e., rigid, see Fig. 13), represents the relative upward frequency-shift between the soft and rigid extremes of material behavior.

The fact that for near-soft (or near-rigid) inclusions the first background lobe of $A_{0}$ (or $A_{1}$ ) occurs before all the others, has useful physical implications. This ordering determines if the inclusions will first oscillate either radially, in-and-out, or translationally back-and-forth. This simple phenomenon has an additive influence on the dispersion curves of wavenumber versus frequency for composites containing many such inclusions. These dispersion curves usually have two branches denoted the acoustical and optical branches [12], by analogy with similar branches observed in (ordered) crystals. Along the acoustical branches the radial motion of the monopole mode, excited by the (always strongest) monopole resonance, is dominant. Along the (higher frequency) optical branches, the translational motion of the dipole mode dominates. Theoretical [7] and experimental [13] studies have already indicated the existence of these branches in random composites, and the dominance of monopole or dipole oscillations along the acoustical or the optical branches, respectively.

\section{CONCLUSION}

It is easier to excite monopole (purely radial) modes of vibration in soft background cases such as that of an epoxy sphere in a steel matrix. For rigid background cases, such as a steel sphere in epoxy, dipole modes are excited first, although with weaker amplitudes. Dipole oscillations are rigid-body translational motions of the inclusion, backand forth, within the matrix. Thus, in this case, the first lobe of the background curve of $A_{1}$ occurs first. As far as the resonances proper are concerned, the fundamental (first) resonance of $A_{0}$ almost always moves up in frequency, and occurs after those of $A_{1}$ and $A_{2}$, provided that the inclusion is solid. If it is fluid, particularly a gas, then the first resonance of $A_{0}$ occurs first, and it is giant. In all cases, all fundamental resonances are within the Rayleigh region $(a / \lambda \approx 1)$.

We have presented the pertinent theory necessary to carry on the resonance scattering analysis of plane elastic waves diffracted by a spherical elastic inclusion in a purely elastic matrix. Losses are present due to scattering and mode-conversion mechanisms. We have applied the formulation to the particular but opposite examples of a plastic inclusion in a metal matrix, and a metallic inclusion in a plastic matrix. This combinations exhibit very different extremes of material behavior. Using the terminology of the RST, one example is dominantly controlled by the soft background, while the other by the rigid. We have displayed the real and the imaginary parts as well as the modulus of the first three (complex) multipole coefficients $A_{n}$, in a wide frequency band for both matrix/inclusion combinations mentioned above. Absorption in the matrix/inclusion composite is controlled within each mode by $\mathrm{Re}$
$\left[A_{n}\right]$. The effective wavespeed is governed by $\operatorname{Im}\left[A_{n}\right]$. Since these quantities have these important physical significances, they were displayed in all instances.

In the standard manner of the RST, we have separated the background from the resonance contributions in all the above cases. This separation clearly displays the interplay between backgrounds and resonances within the multipole coefficients $A_{n}$. This permits us to assess quantitatively the upward frequency shift in the background curves, as well as in the associated resonances, that appears as the inclusion's composition becomes stiffer relative to that of the matrix. It is the upward frequency shift in modal backgrounds that is responsible for the dominance of either radial (monopole) or back-and-forth translational (dipole) oscillations of the inclusion. The first (monopole) case influences the acoustical branches of the dispersion curves [7], [13], while the second affects only the so-called optical branches. The case of gas inclusions studied earlier [7], [11], [14] stands separate from the present case of solid inclusions, since there, the monopole resonance always occurs first and is of gigantic amplitude compared to all others for reasons discussed above and elsewhere [11].

With the methodology of the RST [6], [7] which we have applied here to important examples, one can compute similar curves for all possible material combinations for the matrix and the inclusion, in all frequency bands. This can be done, as was done here, for the case of incident compressional elastic waves and scattered compressional waves. We can also study the case of scattered shear waves. This case involves the use of coefficients $B_{n}$ given in (4), which were not used in the examples in Section III. The resonance scattering theory for incident shear waves and scattered waves of any kind, was first developed elsewhere [6] and requires the use of four additional coefficients $C_{n}, D_{n}, E_{n}$, and $F_{n}$, and numerous other parameters such as resonance widths, etc. Other analogous resonance interaction cases in scattering and in radiating processes have been reviewed recently [15]. Extensions of the present work to the case of many inclusions in the matrix have been also completed recently [16].

\section{APPENDIX \\ The Coefficients $d_{i j}$}

We list here the coefficients $d_{i j}$, which result from the boundary conditions [6], [16]. The spherical Hankel functions referred to here as $h_{n}(x)$ are normally referred to as $h_{n}^{1}(x)$. The media 1 and 2 compression $(d)$ and shear $(s)$ waves are designated by

$$
x_{d i} \equiv k_{d i} a \quad x_{s i} \equiv k_{s i} a, \quad i=1,2 ;
$$

while $\rho_{1}$ and $\rho_{2}$ are the media mass densities:

$$
\begin{aligned}
& d_{11}=x_{d_{1}} h_{n}^{\prime}\left(x_{d_{1}}\right) \\
& d_{21}=h_{n}\left(x_{d_{1}}\right) \\
& d_{31}=\left[2 n(n+1)-x_{s_{1}}^{2}\right] h_{n}\left(x_{d_{1}}\right)-4 x_{d_{1}} h_{n}^{\prime}\left(x_{d_{1}}\right)
\end{aligned}
$$




$$
\begin{aligned}
d_{41}= & x_{d_{1}} h_{n}^{\prime}\left(x_{d_{1}}\right)-h_{n}\left(x_{d_{1}}\right) \\
d_{12}= & n(n+1) h_{n}\left(x_{s_{1}}\right) \\
d_{22}= & x_{s_{1}} h_{n}^{\prime}\left(x_{s_{1}}\right)+h_{n}\left(x_{s_{1}}\right) \\
d_{32}= & 2 n(n+1)\left[x_{s_{1}} h_{n}^{\prime}\left(x_{s_{1}}\right)-h_{n}\left(x_{s_{1}}\right)\right] \\
d_{42}= & {\left[n(n+1)-\frac{1}{2} x_{s_{1}}^{2}-1\right] h_{n}\left(x_{s_{1}}\right)-x_{s_{1}} h_{n}^{\prime}\left(x_{s_{1}}\right) } \\
d_{13}= & -x_{d_{2}} j_{n}^{\prime}\left(x_{d_{2}}\right) \\
d_{23}= & -j_{n}\left(x_{d_{2}}\right) \\
d_{33}= & \frac{-\rho_{2}}{\rho_{1}} \cdot \frac{x_{s_{1}}^{2}}{x_{s_{2}}^{2}}\left\{\left[2 n(n+1)-x_{s_{2}}^{2}\right] j_{n}\left(x_{d_{2}}\right)\right. \\
& \left.-4 x_{d_{2}} j_{n}^{\prime}\left(x_{d_{2}}\right)\right\} \\
d_{43}= & \frac{-\rho_{2}}{\rho_{1}} \cdot \frac{x_{x_{1}}^{2}}{x_{s_{2}}^{2}}\left[x_{d_{2}} j_{n}^{\prime}\left(x_{d_{2}}\right)-j_{n}\left(x_{d_{2}}\right)\right] \\
d_{14}= & -n(n+1) j_{n}\left(x_{s_{2}}\right) \\
d_{24}= & -x_{s_{2}} j_{n}^{\prime}\left(x_{s_{2}}\right)-j_{n}\left(x_{s_{2}}\right) \\
d_{34}= & -2 n(n+1) \frac{\rho_{2}}{\rho_{1}} \frac{x_{s_{1}}^{2}}{x_{s_{2}}^{2}}\left[x_{s_{2}} j_{n}^{\prime}\left(x_{s_{2}}\right)-j_{n}\left(x_{s_{2}}\right)\right] \\
d_{44}= & \frac{-\rho_{2}}{\rho_{1}} \frac{x_{s_{1}}^{2}}{x_{s_{2}}^{2}}\left\{\left[n(n+1)-\frac{1}{2} x_{s_{2}}^{2}-1\right] j_{n}\left(x_{s_{2}}\right)\right. \\
& \left.-x_{s_{2}} j_{n}^{\prime}\left(x_{s_{2}}\right)\right\},
\end{aligned}
$$

The first and second columns $\left(d_{i 1}\right.$ and $\left.d_{i 2}\right)$ are complex and depend exclusively on the external medium's compression $\left(x_{d_{1}}\right)$ and shear waves $\left(x_{s_{1}}\right)$, respectively. The third and fourth columns $\left(d_{i 3}\right.$ and $\left.d_{i 4}\right)$ are real and predominantly functions, respectively, of the compression $\left(x_{d_{2}}\right)$ and shear waves $\left(x_{s_{2}}\right)$ within the sphere.

\section{REFERENCES}

[1] C. F. Ying and R. Truell, "Scattering of a plane longitudinal wave by a spherical obstacle in a isotropically elastic solid," J. Appl. Phys., vol. 27, pp. 1086-1097, 1956.

[2] Y. H. Pao and C. C. Mow, "Scattering of a plane compressional wave by a spherical obstacle," J. Appl. Phys., vol. 34, pp. 439-499, 1963.

[3] R. Truell et al., Ultrasonic Methods in Solid State Physics. New York: Academic, 1969 , ch. 3

[4] Y. H. Pao and C. C. Mow, Diffraction of Elastic Waves and Dynamic Stress Concentrations. New York: Crane Russak, 1971, ch. 6.

[5] L. Flax, G. Gaunaurd, and H. Ueberall, "The theory of resonance scattering," in Physical Acoustics, vol. 15, W. P. Mason and R. N. Thurston, Eds. New York: Academic, 1981, ch. 3, pp. 191-294.

[6] D. Brill and G. Gaunaurd, "Resonance theory of elastic waves ultrasonically scattered from an elastic sphere," J. Acoust. Soc. Amer., vol. 81, pp. 1-21, 1987.

[7] G. Gaunaurd and H. Ueberall, "Resonance effects and the ultrasonic effective properties of particulate composites," J. Acoust. Soc. Amer., vol. 74, pp. 305-313, 1983.
[8] G. Gaunaurd, "Multipole resonances in elastic wave-scattering from cavities and in acoustic wave-scattering from bubbles and droplets," in Mathematical Methods and Applications of Scattering Theory, A. Sáenz, Ed. Springer-Verlag, 1979, pp. 114-120.

[9] G. Gaunaurd and C. Tsui, "Transient and steady-state target-resonance excitation by sound scattering," J. Appl. Acoust, vol. 23, pp. $121-140,1988$

[10] G. C. Gaunaurd and A. Kalnins, "Resonances in the sonar crosssections of (coated) spherical shells," Int. J. Solids Structures, vol. 18, pp. 1083-1102, 1982.

[11] G. Gaunaurd et al., "Giant monopole resonances in the scattering of waves from gas-filled spherical cavities and bubbles," J. Acoust. Soc. Amer., vol. 65, pp. 573-594, 1979.

[12] F. C. Moon and C. C. Mao, "Wave propagation in a composite material containing dispersed rigid spherical inclusions," Rand Corp., Rand, Santa Monica, Rep. RM-6139-PR, 1970.

[13] V. K. Kinra and A. Anand, "Wave propagation in a random particulate composite at short and long wave-lengths," Int. J. Solids Structures, vol. 18, pp. 367-380, 1982.

[14] E. Meyer, K. Brendel, and K. Tamm, "Pulsation oscillations of cavities in rubber," J. Acoust. Soc. Amer., vol. 30, pp. 1116-1124, 1958.

[15] D. Brill, V. Ayres, and G. Gaunaurd, "The influence of natural resonances in radiation and scattering processes," J. Washington Acad. Sci.

[16] G. Gaunaurd and W. Wertman, "Comparison of effective medium theories for inhomogeneous continua," J. Acoust. Soc. Amer, vol. 84,1988 , to be published.

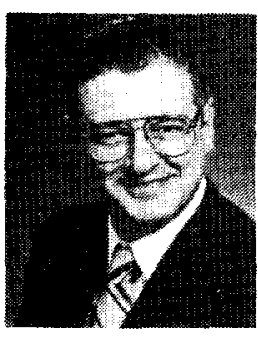

Guillermo C. Gaunaurd (M'82-SM'83) was born July 19, 1940 in Havana, Cuba. He received the $\mathrm{Ph} . \mathrm{D}$. degree in acoustics from Catholic University, Washington, DC, in 1971 .

He has been a Research Physicist at the Research Department of the Naval Surface Weapons Center, White Oak, MD, since 1971, specializing in acoustics and electromagnetic radiation and scattering. He is currently Group Leader in the Electromagnetics Branch of that institution where he carries on and supervises a research program on the basic interaction of electromagnetic, acoustic, and elastic waves with materials and structures. Since 1978 he has occasionally Lectured at Catholic University and since 1984 at the School of Engineering of the University of Maryland, College Park.

Dr. Gaunaurd is a member of the New York Academy of Sciences, the American Physical Society, the Philosophical Society of Washington, the ASME, the American Academy of Mechanics, Sigma Xi, Tau Beta Pi, the International Union of Mathematical Physies, and others. He is a Fellow of the Acoustical Society of America and of the Washington Academy of Sciences. He has been a member of several technical committees in various professional societies. He had authored over 100 scientific journal articles and 30 government technical reports. He has given over 130 technical presentations before professional societies or at congress/symposia. He holds four patents and has authored four review chapters on his own work. His professional biography appears in American Men and Women of Science, Marquis" Who's Who, and other similar books.

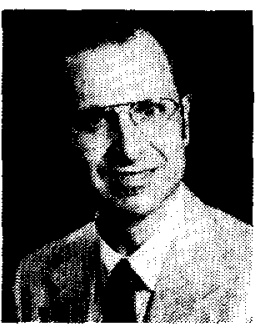

William H. Wertman was born in Washington, DC, on December 31, 1937. He received the A.B. degree in mathematics from Gettysburg College, Gettysburg, PA, and the M.S. degree from Lehigh University, Bethlehem, PA, in 1960 and 1962, respectively.

In 1962 he joined the Naval Surface Weapons Center as a Mathematician. He is also an Adjunct Professor at Montgomery College. His special interests include computer simulation of nonlinear processes and computer graphics.

Mr. Wertman is a member of the Mathematical Association of America and is the author of numerous technical reports relating to mine warfare. 